https://helda.helsinki.fi

\title{
TPB and the invasion of adaptive dynamics
}

\author{
Kisdi, Eva
}

2020-06

Kisdi , E 2020 , ' TPB and the invasion of adaptive dynamics ' , Theoretical Population Biology , vol. 133 , pp. 52-55 . https://doi.org/10.1016/j.tpb.2019.12.003

http://hdl.handle.net/10138/323370

https://doi.org/10.1016/j.tpb.2019.12.003

cc_by_nc_nd

acceptedVersion

Downloaded from Helda, University of Helsinki institutional repository.

This is an electronic reprint of the original article.

This reprint may differ from the original in pagination and typographic detail.

Please cite the original version. 


\section{TPB and the invasion of adaptive dynamics}

Eva Kisdi

Department of Mathematics and Statistics

University of Helsinki, Finland

email: eva.kisdi@helsinki.fi

This article has been published. This is the accepted manuscript (post peer review, pre-copyedit version) of a commentary published in the $50^{\text {th }}$ anniversary issue of Theoretical Population Biology.

Please cite the article in the journal: Kisdi, E. 2020. TPB and the invasion of adaptive dynamics. Theor. Pop. Biol. 133: 52-55. https://doi.org/10.1016/j.tpb.2019.12.003.

In the past two decades, adaptive dynamics has become a widely used framework to understand evolution as driven by ecological interactions (Geritz et al. 1998). This is a theory of evolution rooted firmly in ecology, born at a time when, despite efforts in game theory and life history optimisation, evolution was generally considered to be on the turf of population genetics. Facing the traditional divide between these fields, one could learn to appreciate that Theoretical Population Biology (TPB) houses population genetics and population ecology under one roof! In this commentary, I congratulate TPB on its $50^{\text {th }}$ anniversary by highlighting a few personal favourites from the journal's history with adaptive dynamics.

Adaptive dynamics derives natural selection from population dynamics, via explicitly modelling the growth of different strains or alleles. To investigate the long-term evolution of quantitative traits, it assumes that mutations are sufficiently rare so that the population equilibrates before the next mutation appears. Evolution can then be seen as a sequence of mutations that invade and either replace the former strain or allele or coexist with it, as governed by the joint population dynamics of the initial resident and the invading mutant (note however that the results are robust with respect to the frequency of mutations, see M eszéna et al. 2005). Assuming also that each mutation has only a small effect on the trait value, the dynamics of trait values becomes smooth and deterministic, and exhibits phenomena such as diversification by evolutionary branching (Geritz et al. 1998; see Box 1 for key definitions). 


\section{Forerunners}

Astronomers are familiar with what they call pre-discovery sightings, celestial bodies recorded on photographs before it is realized that these dots are the same object. In a somewhat similar vein, results in specific models prefigured the general theory of adaptive dynamics. Two papers by Christiansen and Loeschcke (1980; 1987), published in TPB, stand out in particular ${ }^{1}$. Their model investigated the evolution of a resource utilisation trait, assuming alleles with similar phenotypic effects. In the first paper, they showed that the allele closest to the resource optimum (i.e., to the trait that today would be called singular) goes to fixation if the optimum is outside the range of the standing genetic variation; if it is inside, then two alleles remain, and these are the outermost two alleles if the resource spectrum is broad. It is easy to see convergence stability in the first result and disruptive selection at an evolutionary branching point in the second. M oreover, Figures 1 and 2 of Christiansen and Loeschcke (1980) are essentially pairwise invasibility plots (PIPs; Figure 1). PIPs have become iconic figures of adaptive dynamics, but they were in sporadic use also before, as invasibility analysis gained momentum. M otro (1982), writing on evolutionarily stable dispersal strategies, showed another early example of PIPs in TPB.

Christiansen and Loeschcke (1987) went beyond all forerunners in extending their model to multivariate (vector-valued) traits, to find that the maximum level of polymorphism near the resource optimum increases with the dimensionality of the trait space. One usually pictures diversification as an ancestral line splitting into two lineages. M ight the polymorphism result of Christiansen and Loeschcke (1987) indicate evolutionary branching into three or more diverging lineages, instead of the usual two? It took nearly 30 years to learn that the answer is no (Geritz et al. 2016).

\section{Different types of stability}

From the 1980 's, there was growing awareness that evolutionary stability is different from stability in the usual dynamical sense. In other words, whether a strategy is uninvadable (called an evolutionarily stable strategy, ESS) has little to do with whether the evolving trait values are attracted to it (now called convergence stability; see also Van Cleve in this issue). Eshel and colleagues were keen on finding "continuously stable strategies", which possess both kinds of stability (Eshel and M otro 1981 in TPB; see also Eshel 1983; Eshel et al. 1997). Could the two stability

\footnotetext{
${ }^{1} A$ third paper in the same series, Loeschcke and Christiansen (1984), links their work to multilocus population genetics and is discussed by Bürger (in this issue).
} 
properties combine freely? Two quotes from TPBillustrate the struggle with this question. One opinion dismissed non-attracting ESSs, restricting the analysis to attractors: "provided that an ESS exists, we should be able to find it using strategy dynamics" (Vincent et al. 1993). And the opposite: "[it is] easy to provide mathematical examples of functions with equilibrium points which are $\mathrm{m}$ stable but not $\delta$-stable [i.e., convergence stable but not evolutionarily stable], but I have yet to see a biologically plausible example of this" - Taylor (1989) was unfortunate to miss the evolutionary branching points!

Taylor's (1989) paper nevertheless stands out for connecting convergence stability to kin selection theory (reviewed by Van Cleve in this issue). Vincent et al. (1993), together with Brown and Vincent (1987) also in TPB, belong to the start of an alternative framework of adaptive dynamics, now less in use, with its own concept of evolutionary stability. Whereas the framework of Geritz et al. (1998) requires only local stability thus making use of convenient analytical tools based on local expansions, Vincent and co-workers took the "safer" definition that an ESS must be globally stable against the invasion of any mutant trait, not only against mutations of small effect.

\section{Polymorphisms and bifurcations}

Adaptive dynamics is particularly well suited to studying the evolution of diversity via evolutionary branching (Geritz et al. 1998). An early paper on evolutionary branching in TPB is Geritz et al. (1999), who investigated seed size evolution driven by competition for living sites. M any other analyses stop at detecting the first branching point, i.e., at demonstrating that an initially monomorphic population evolves to become dimorphic. In contrast, Geritz et al. (1999) put much emphasis on how the dimorphic population branches further and how the population evolves at higher levels of polymorphism. This example also highlights that evolutionary branching is neither a necessary nor a sufficient condition for long-term polymorphism; new branches of the evolutionary tree may subsequently go extinct, and polymorphic ESSs may exist but be unreachable via the gradual process of evolutionary branching.

Further, Geritz et al. (1999) laid out the basics of bifurcation theory for adaptive dynamics. Similarly to attractors in dynamical systems, evolutionary branching points can undergo fold bifurcations. M ore interestingly, a convergence stable ESS can lose its evolutionary stability without losing its convergence stability, whereby it becomes an evolutionary branching point; this bifurcation has no analogue in classic dynamical systems. Once the ESS has become a branching point, the population 
will become dimorphic, but how far will the two branches separate? A continuity argument might suggest that close to the bifurcation, evolutionary branching stops with a slight separation. Geritz et al. (1999) showed that this is not so. The ESS-branching point bifurcation implies an abrupt ("catastrophic") change, so that the two branches typically evolve out of the neighbourhood of the branching point however close the system is to the bifurcation. The reason is that a dimorphic ESS typically exists already before the monomorphic ESS would bifurcate, so that upon the bifurcation, the population can evolve to this pre-existing attractor. The birth of the dimorphic attractor is connected to the loss of global, not local, evolutionary stability. Populations thus often have multiple ESSs with different numbers of coexisting strains, and there is an intimate link between the evolutionary dynamics at different levels of polymorphism.

Both convergence stability and evolutionary stability depend on the trade-offs that link the demographic parameters with the evolving trait. Changing the shape of a trade-off function readily generates bifurcations; roughly speaking, a trade-off strongly favouring a generalist results in an ESS, whereas a trade-off strongly favouring the extreme phenotypes produces a repellor. For evolutionary branching, the trade-off must pass in between. How broad is the range of evolutionary branching, and is there such a range to start with? Simple models may give explicit conditions for evolutionary branching, but this is the exception; in more complex models, one must fall back on numerical analysis. The traditional approach is then to specify the trade-off as some parameterised function and perform the bifurcation analysis in terms of its parameters (e.g. Geritz et al. 1999). However, the choice of the trade-off functions is usually ad hoc, because experimental data do not constrain the trade-offs with sufficient precision, and because models deriving the trade-offs are lacking. Here critical function analysis offers a way out (de Mazancourt and Dieckmann 2004; Kisdi 2006, 2015). This method constructs the most extreme trade-off functions in between which the model yields a certain prediction, capitalizing on the fact that evolutionary branching points and other evolutionary singularities depend only on local properties of the trade-off function, which can be parameterised without choosing the shape of the function globally. In TPB, Geritz et al. (2007) used this technique for the first time, in a model where previous analysis with a specific trade-off function found no evolutionary branching. The model is not accessible for mathematical analysis because the population dynamic attractor is a limit cycle, which cannot be obtained explicitly; yet Geritz et al. (2007) proved that evolutionary branching is possible and characterised the trade-off functions that lead to evolutionary branching. 


\section{The dark side: Evolutionary suicide}

In stark contrast to diversification, adaptive dynamics also predicts that species can evolve to their own extinction. Evolutionary suicide occurs when the evolving trait passes through a catastrophic bifurcation of population dynamics, whereby the population crashes to extinction (Gyllenberg and Parvinen 2001; reviewed in TPB by Dieckmann and M etz 2006). Note the difference between suicide and murder: In polymorphic populations, the evolution of one strain can push another to extinction (as in Geritz et al. 1999 discussed above), but in evolutionary suicide, natural selection in the focal strain leads to its own demise.

The first example of evolutionary suicide I am aware of was published in TPB, by M atsuda and Abrams (1994). Though they did not use the name, they described the phenomenon in a simple, transparent model of a prey exploited by a fixed number of predators. If all prey individuals are active and thus accessible for the predators, then the predator functional response is saturated and the per capita risk of being killed is low (to put it simply, I am safe because the predators are busy eating others). Low per capita mortality coupled with the high fecundity of active foragers ensures that the prey population equilibrates at a high density, maintaining predator saturation. However, natural selection can still favour prey with a less active lifestyle, hiding from the predator instead of foraging. For an active individual, the risk of attack increases when the others are hiding, because then the predators are less saturated; and for the population, the equilibrium density decreases. In turn, the higher predation risk further selects the prey to hide rather than forage. A vicious circle thus starts as more and more predators are free to search for fewer and fewer active prey, ever increasing the selection pressure towards hiding. Eventually, the prey population becomes too inactive for reproduction to balance mortality. As population size falls, the risk of predation further increases, leading to extinction through a fold bifurcation of prey density.

\section{A probabilist's view}

Evolution ultimately depends on the appearance of random mutations. In a finite world, the ecological dynamics are also stochastic. By taking appropriate limits of large population size and infrequent small mutations (M etz et al. 1996), however, adaptive dynamics retains only one significant source of randomness, the demographic stochasticity of new mutants. New mutations are initially present in a single copy, and their invasion probabilities influence evolution as described by the canonical equation of adaptive dynamics (Dieckmann and Law 1996; Durinx et al. 2008; see Box 
1). Different limits of the demographic rates and of the size and frequency of mutations, however, yield very different evolutionary models, including deterministic and stochastic integro-differential equations and diffusion processes. Champagnat et al. (2006) gave an excellent summary of these in TPB, reviewing also the authors' previous work on the probabilistic foundation of adaptive dynamics.

Finite population size is an obvious obstacle to diversification, as genetic drift tends to eliminate variation. Under disruptive selection, however, diversification can increase population size, which makes further diversification more likely; and conversely, low diversity implies small population size, hindering diversification. Debarre and Otto (2016) showed in TPBa regime of bistability, with stochastic oscillations between states of high and low variance, which separates populations too small to undergo evolutionary branching from those large enough to branch as predicted by the deterministic theory of adaptive dynamics.

\section{Coexistence}

The question of diversification is intimately connected with the question of coexistence. In particular, the theory of limiting similarity appears to be in conflict with evolutionary branching. The theory of limiting similarity posits that coexisting strains or species must have sufficiently differentiated traits, so that they use different resources or fill different niches, to escape competitive exclusion. If similar strains cannot coexist, how can diversity evolve through initially small differences? The answer is in a landmark paper in TPB, where M eszéna et al. (2006, see also Barabás et al. 2014) showed that limiting similarity is a problem of robustness rather than of existence. They showed that a coexistence equilibrium exists for any strains, however similar; but only for a narrow set of ecological parameters. The ecological parameters include the evolving traits themselves. In agreement with limiting similarity, a given pair of similar strains will not coexist unless the environment is fine-tuned for coexistence (which is unlikely to happen in nature). However, in any given environment, the adaptive dynamics of the traits can lead to those specific values, i.e., to the evolutionary singularities, where coexistence and evolutionary branching are possible.

In the parallel world of population genetics, limiting similarity went under disguise. Classic models of multiple niche polymorphism predicted that genetic variability can be maintained if different alleles are favoured in different niches such as different subpopulations, but the same models found lack of robustness under weak selection (e.g. Hoekstra et al. 1985; see also Bürger in this issue). Since selection is necessarily weak if the alleles have similar effects, this result directly corresponds to 
limiting similarity sensu M eszéna et al. (2006). Evolutionary branching can nevertheless produce multiple niche polymorphisms robustly, by first evolving the allelic effects to the point where the conditions for polymorphism are just right (Kisdi and Geritz 1999).

For half a century, ecologists and population geneticists used models related to multiple niche polymorphism to study how spatial and temporal variability can maintain diversity. Using adaptive dynamics, this work has recently been synthesized and vastly expanded to allow for arbitrary patterns of spatiotemporal variation by Svardal et al. (2015), the first winner of TPB's Feldman Prize (announced in Rosenberg 2018).

\section{Beyond invasion}

Adaptive dynamics has invaded the scientific landscape as a powerful tool to study evolution by natural selection (see https://www.mv.helsinki.fi/home/ kisdi/addyn.htm for a wider selection of adaptive dynamics papers, including many more in TPB). Where does this invasion lead to? Adaptive dynamics will not obey one of its own theorems, usually paraphrased as invasion implies fixation (Geritz 2005), because it is not a small mutation away from other conceptual frameworks used to model evolution. On the one hand, adaptive dynamics simplifies ruthlessly by assuming clonal inheritance instead of taking on the intricacies of multilocus population genetics (see Bürger in this issue), and while it investigates diversification, it usually ignores the question of reproductive isolation leading to speciation. On the other hand, adaptive dynamics takes ecology seriously, and derives natural selection directly from differential population growth in possibly complex ecological systems. Doing so has led us to realize that selection is frequency dependent as a rule. This implies rich dynamics and explains how ecological interactions promote the evolution of diversity, including diversity that may be conducive to speciation.

While adaptive dynamics offers a handy toolkit to analyse concrete models of interest, much is still to be done especially for multidimensional and function-valued traits. For evolutionary branching in higher-dimensional trait spaces, only sufficient (but not necessary) conditions are known (Geritz et al. 2016). For function-valued traits, the canonical equation has been derived ( $M$ etz et al. 2016), but strong convergence stability (see Box 1 ) and evolutionary branching are open problems. 
Acknowledgements. I am grateful for the editorial comments of Noah Rosenberg and I acknowledge financial support from the Academy of Finland through the Centre of Excellence in Analysis and Dynamics Research.

\section{References}

Barabás Gy., G. M eszéna \& A. Ostling. 2014. Fixed point sensitivity analysis of interacting structured populations. Theor. Pop. Biol. 92: 97-106.

Brown J.S. \& T. L. Vincent. 1987. A theory for the evolutionary game. Theor. Pop. Biol. 31: 140-166.

Bürger R. 2020. M ultilocus population-genetic theory. Theor. Pop. Biol., this issue.

Champagnat N., R. Ferriere \& S. M éléard. 2006. Unifying evolutionary dynamics: From individual stochastic processes to macroscopic models. Theor. Pop. Biol. 69: 297-321.

Christiansen F. B. \& V. Loeschcke. 1980. Evolution and intraspecific exploitative competition. I. One locus theory for small additive gene effects. Theor. Pop. Biol. 18: 297-313.

Christiansen F. B., V. Loeschcke. 1987. Evolution and intraspecific competition III. One-locus theory for small additive gene effects and multidimensional resource qualities. Theor. Pop. Biol. 31: 33-46.

Debarre F. \& S. P. Otto. 2016. Evolutionary dynamics of a quantitative trait in a finite asexual population. Theor. Pop. Biol. 108: 75-88.

Dieckmann U. \& R. Law. 1996. The dynamical theory of coevolution: A derivation from stochastic ecological processes. J. Math. Biol. 34: 579-612.

Dieckmann U. \& J. A. J. M etz. 2006. Surprising evolutionary predictions from enhanced ecological realism. Theor. Pop. Biol. 69: 263-281.

Durinx M. . J. A. J. M etz \& G. Meszéna. 2008. Adaptive dynamics for physiologically structured population models. J. Math. Biol. 56: 673-742.

Eshel I. 1983. Evolutionary and continuous stability. J. theor. Biol. 103: 99-111.

Eshel I. \& U. M otro. 1981. Kin selection and strong evolutionary stability of mutual help. Theor. Pop. Biol. 19: 420-433.

Eshel I., U. M otro \& E. Sansone. 1997. Continuous stability and evolutionary convergence. J. theor. Biol. 185: 333-343.

Geritz S. A. H. Resident-invader dynamics and the coexistence of similar strategies. J. Math. Biol. 50: 67-82.

Geritz S. A. H., E. Kisdi, G. M eszéna \& J. A. J. Metz. 1998. Evolutionarily singular strategies and the adaptive growth and branching of the evolutionary tree. Evol. Ecol. 12: 35-57. 
Geritz S. A. H., E. Kisdi \& P. Yan. 2007. Evolutionary branching and long-term coexistence of cycling predators: Critical function analysis. Theor. Pop. Biol. 71: 424-435.

Geritz S. A. H., E. van der M eijden \& J. A. J. Metz. 1999. Evolutionary dynamics of seed size and seedling competitive ability. Theor. Pop. Biol. 55: 324-343.

Geritz S. A. H., J. A. J. M etz \& C. Rueffler. 2016. Mutual invadability near evolutionarily singular strategies for multivariate traits, with special reference to the strongly convergence stable case. J. Math. Biol. 72: 1081-1099.

Gyllenberg M. \& K. Parvinen. 2001. Necessary and sufficient conditions for evolutionary suicide. Bull. Math. Biol. 63: 981-993.

Hoekstra R. F., R. Bijlsma \& J. Dolman. 1985. Polymorphism from environmental heterogeneity: Models are only robust if the heterozygote is close in fitness to the favoured homozygote in each environment. Genet. Res. Camb. 45: 299-314.

Kisdi E. 2006. Trade-off geometries and the adaptive dynamics of two co-evolving species. Evol. Ecol. Res. 8: 959-973.

Kisdi É. 2015. Construction of multiple trade-offs to obtain arbitrary singularities of adaptive dynamics. J. Math. Biol. 70: 1093-1117.

Kisdi E. \& S. A. H. Geritz. 1999. Adaptive dynamics in allele space: Evolution of genetic polymorphism by small mutations in a heterogeneous environment. Evolution 53: 993-1008.

Loeschcke V. \& F. B. Christiansen. 1984. Evolution and intraspecific exploitative competition. II. A two-locus model for additive gene effects. Theor. Pop. Biol. 26: 228-264.

de Mazancourt C. \& U. Dieckmann. 2004. Trade-off geometries and frequency-dependent selection. Am. Nat. 164: 765-778.

Matsuda H. \& P. A. Abrams. 1994. Timid consumers: Self-extinction due to adaptive change in foraging and anti-predator effort. Theor. Pop. Biol. 45: 76-91.

M eszéna G., M. Gyllenberg, F. J. Jacobs \& J. A. J. M etz. 2006. Link between population dynamics and dynamics of Darwinian evolution. Phys. Rev. Letters 95: 078105.

Meszéna G., M. Gyllenberg, L. Pásztor \& J. A. J. M etz. 2006. Competitive exclusion and limiting similarity: A unified theory. Theor. Pop. Biol. 69: 68-87.

Metz, J. A. J., S. A. H. Geritz, G. M eszéna, F. J. A. Jacobs, and J. S. van Heerwaarden. 1996. Adaptive dynamics, a geometrical study of the consequences of nearly faithful reproduction. In: S. J. van Strien, and S. M. Verduyn Lunel (eds.): Stochastic and spatial structures of dynamical systems. North Holland, Amsterdam, The Netherlands, pp. 183-231.

M otro U. 1982. Optimal rates of dispersal. I. Haploid populations. Theor. Pop. Biol. 21: 394-411.

Rosenberg N. 2018. Editorial: The 2018 M arcus W. Feldman Prize in Theoretical Population Biology. Theor. Pop. Biol. 119: 1-2. 
Svardal H., C. Rueffler \& J. Hermisson. 2015. A general condition for adaptive genetic polymorphism in temporally and spatially heterogeneous environments. Theor. Pop. Biol. 99: 76-97.

Taylor P. D. 1989. Evolutionary stability in one-parameter models under weak selection. Theor. Pop. Biol. 36: 125-143.

Van Cleve J. 2020. Synthesis in evolutionary game theory, kin selection, and population genetics. Theor. Pop. Biol., this issue

Vincent T. L., Y. Cohen \& J. S. Brown. 1993. Evolution via strategy dynamics. Theor. Pop. Biol. 44: 149-176. 


\section{Box 1. Key definitions in adaptive dynamics}

Invasion fitness: $s_{x}\left(x_{m u t}\right)$ is the long-term, time-averaged exponential growth rate of a mutant strain with trait value $x_{\text {mut }}$ in the resident population of strain $x$. The trait value may be a scalar or a vector. Since the resident strain has zero long-term growth, $s_{x}(x)=0$.

Pairwise invasibility plot (PIP): sign plot of $s_{x}\left(x_{m u t}\right)$ for scalar-valued traits (see Figure 1).

(a)

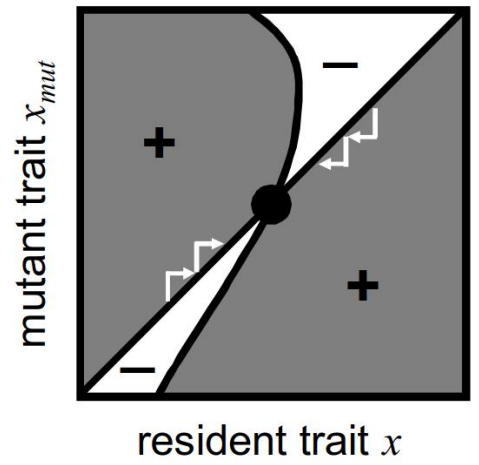

(b)

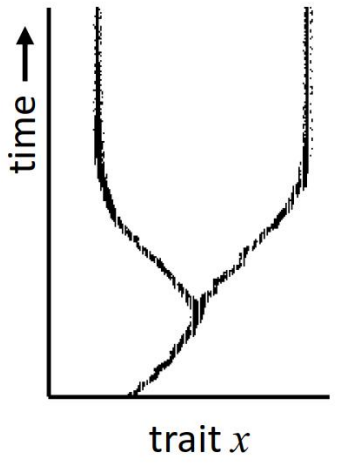

Figure 1. Examples of key graphics in adaptive dynamics. (a) Pairwise invasibility plot (PIP). In the dark-shaded areas marked with " + ", the invasion fitness is positive and therefore the mutant can invade; in the unshaded areas marked with "-", the mutant dies out. The white arrows indicate evolution by invasion and substitution of mutants, and the filled circle marks the singularity. In this figure, the singularity is convergence stable (arrows) but not evolutionarily stable ("+" above and below the singularity shows that it can be invaded), i.e., it is an evolutionary branching point. This figure is a schematic illustration. (b) Evolutionary branching as seen in a simulation (based on the haploid version of the Levene model in Kisdi and Geritz 1999).

Selection gradient: $D(x)=\left[\partial s / \partial x_{m u t}\right]_{x m u t=x}$. To first order, invasion fitness is given by

$s_{x}\left(x_{m u t}\right)=D(x)\left(x_{m u t}-x\right)+O\left(\left(x_{m u t}-x\right)^{2}\right)$ so that, for scalar traits, a mutant with trait value higher (lower) than the resident can invade if the selection gradient is positive (negative).

Singularity: a trait value $x^{*}$ is singular if $D\left(x^{*}\right)=0$. 
Invasion implies fixation theorem: Away from singularities and assuming mutations of small effect, invasion implies that the mutant spreads to fixation and thus substitutes the former resident (Geritz 2005). By repeated mutations and substitutions, scalar traits evolve in the direction of the selection gradient.

Convergence stability for scalar traits: a singularity $x^{*}$ is convergence stable if a sequence of invasion-substitution events starting from its neighbourhood leads closer to $x^{*}$; this is the case if $D^{\prime}\left(x^{*}\right)<0$.

Evolutionary stability: a singularity $x^{*}$ is evolutionarily stable (ESS) if no mutation of small effect can invade a resident population with trait $x^{*}$; this is the case if $\left[\partial^{2} s / \partial x_{m u t}^{2}\right]_{x m u t=x=x^{*}}<0$ (scalar traits) or if the Hessian matrix is negative definite (vector-valued traits).

Evolutionary branching point for scalar traits: a singularity that is convergence stable but not evolutionarily stable (Geritz et al. 1998; see Figure 1).

Canonical equation of adaptive dynamics: trait dynamics in the limit of infinitesimally small mutations, given by $\dot{x}=K(x) D(x)$ (Dieckmann and Law 1996; Durinx et al. 2008). The positive speed constant $K(x)$ depends on the rate and size of mutations and on the demography of the resident population; to first order in mutation effect size, the probability that an advantageous mutation invades in the face of demographic stochasticity is proportional to $D(x)$. For vector-valued traits, the selection gradient is a vector and $K(x)$ is a positive definite matrix, which depends on the covariances between the effects of a mutation on the elements of the trait vector.

Strong convergence stability for vector-valued traits: the singularity $x^{*}$ is an asymptotically stable fixed point of the canonical equation irrespective of the choice of the positive definite matrix $K\left(x^{*}\right)$, i.e., irrespective of the (usually poorly known) mutational covariances. 